\title{
A NONDESUSPENSION THEOREM FOR STUNTED REAL PROJECTIVE SPACES ${ }^{1}$
}

\author{
DONALD M. DAVIS AND MARK MAHOWALD
}

ABSTRACT. For certain stunted projective spaces $\boldsymbol{P}$ and positive integers $\boldsymbol{n}$, it is shown that $\boldsymbol{P}$ cannot be homotopy equivalent to the $\boldsymbol{n}$-fold suspension of some other complex.

Let $P_{m}^{m+a}=R P^{m+a} / R P^{m-1}$. The purpose of this note is to prove

THEOREM 1. If $\left(\begin{array}{c}m-1 \\ a\end{array}\right)$ is odd and $m \equiv 0(8), P_{m}^{m+a}$ is not an $(m-a+2 \nu(m)$ $+\Delta)$-fold suspension, where $\nu\left(2^{\alpha} \cdot\right.$ odd $)=\alpha$ and $\Delta$ is defined by the table

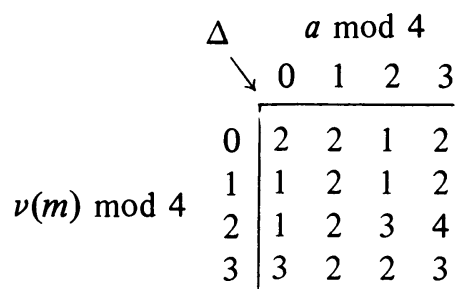

Since $P_{m}^{m+a}$ is the Thom complex of $m \xi_{a}$, where $\xi$ denotes the Hopf bundle over $P^{a}$, this implies

CoRollaRy 2. If $\left(\begin{array}{c}m-1 \\ a\end{array}\right)$ is odd and $m \equiv 0(8), m \xi_{a}$ does not have $(m-a+$ $2 \nu(m)+\Delta)$ linearly independent sections.

Corollary 2 has been proved by several other methods ([2], [3], [4], [5]), all of which involve the Adams operation $\psi^{3}-1$, as does our proof of Theorem 1. However the nondesuspension result is new. Corollary 2 is in some cases slightly weaker than the result of [2]. This suggests the possibility that for some of the strongest nonsectioning theorems, the corresponding nondesuspension result does not hold. For example the methods of this paper do not enable us to prove the nonexistence of $\Sigma^{-19} P_{64}^{120}, \Sigma^{-19} P_{32}^{54}$, or $\Sigma^{-17} P_{48}^{88}$, despite the fact that $64 \xi_{56}, 32 \xi_{22}$, and $48 \xi_{40}$ do not have respectively 19,19 , and 17 sections.

The method of proof is very similar to that of [6]. If $\Sigma^{-(m-a+r)} P_{m}^{m+a}$ exists, we can form the commutative diagram

Received by the editors March 29, 1977.

AMS (MOS) subject classifications (1970). Primary 55D40, 55D35, 55G40, 55G25.

Key words and phrases. Suspension, projective space, geometric dimension, Adams operations.

${ }^{1}$ This work was supported by National Science Foundation, research grant MCS 76-04421. 


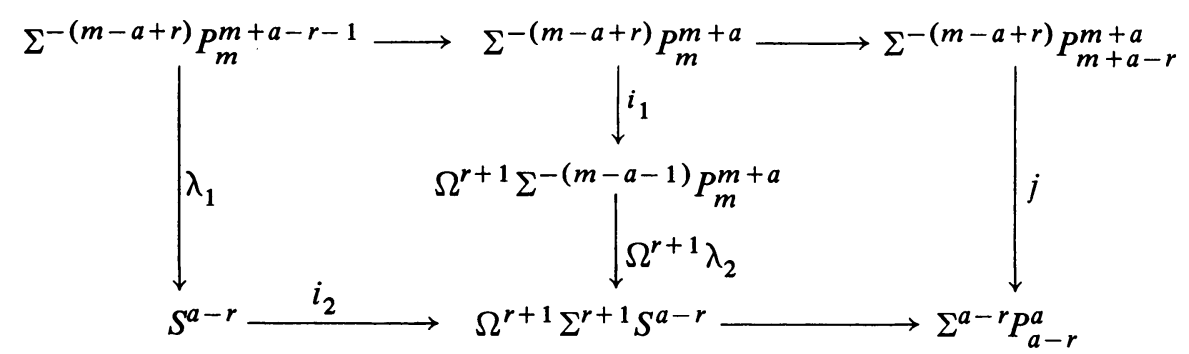

The cofibre of $i_{2}$ is $\Sigma^{a-r} P_{a-r}^{a}$ through dimension $3(a-r)-1$ by $[9,11.7] . j$ is the induced map of cofibres. $\lambda_{1}$ and $\lambda_{2}$ are desuspensions of the map $\lambda$ in the cofibration sequence

$$
S^{m-1} \rightarrow P_{m-1}^{m+b} \rightarrow P_{m}^{m+b} \stackrel{\lambda}{\rightarrow} S^{m}
$$

These desuspensions exist by the Freudenthal suspension theorem.

The mapping cone of $\lambda_{2}$ is $\Sigma^{-(m-a-2)} P_{m-1}^{m+a}$ in which $\mathrm{Sq}^{a}$ is nonzero on the bottom class (since $\left(\begin{array}{c}m-1 \\ a\end{array}\right)$ is odd). Thus by Proposition 5 below, $j^{*}$ sends the next-to-top class nontrivially. The action of $\mathrm{Sq}^{1}$ and $\mathrm{Sq}^{2}$ implies that $j^{*}$ is nontrivial in all dimensions except possibly the top if it is $\equiv 3$ (4) or the bottom if it is $\equiv 0$ (4). Thus the Atiyah-Hirzebruch spectral sequence [3] implies that $j$ and its $S$-dual induce isomorphisms in $\widetilde{K O}$-theory, for $a-r=$ $a-(2 \nu(m)+\Delta) \neq 0(4)$ in Theorem 1 .

By using the Adams operations as computed in [1], we will obtain a contradiction to the existence of $j$ and hence of $\Sigma^{-(m-a+r)} P_{m}^{m+a}$ when $r=2 \nu(m)+\Delta$. Letting $G_{1}$ and $G_{2}$ be generators of $\widetilde{K O}\left(\Sigma^{m} P_{a-r}^{a}\right)$ and $K O\left(P_{m+a-r}^{m+a}\right)$ and $j^{\prime}=\Sigma^{m-a+r} j$, we have $G_{2}=\psi^{3} j^{\prime} G_{1}=j^{\prime} \psi^{3} G_{1}=3^{m / 2} G_{2}$, and hence by [1, 7.4 and 8.1]

$$
\nu(m)+1 \geqslant \varphi^{\prime}(m+a-r, m+a)
$$

where $\varphi^{\prime}(\alpha, \beta)$ is the number of integers $i \equiv 0,1,2,4(8)$ such that

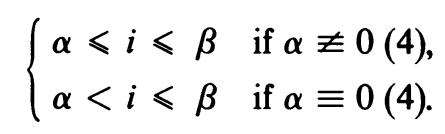

Since $m \equiv 0(8), \varphi^{\prime}(m+a-r, m+a)=\varphi^{\prime}(a-r, a)$. The same argument applied to the Spanier-Whitehead dual of $j$ yields

$$
\nu(m)+1 \geqslant \varphi^{\prime}(-a-1, r-a-1) .
$$

One readily checks that (3) or (4) is contradicted when $r=2 \nu(m)+\Delta$, establishing Theorem 1.

Finally we prove the following proposition, which was used above, relating functional cohomology operations and mappings of loop spaces. For any space $X$ let $\sigma: H^{i}(X) \rightarrow H^{i-1}(\Omega X)$. Let $g_{n}$ be the nonzero class in $H^{n}\left(S^{n}\right)$. All cohomology groups have $\mathbf{Z}_{2}$-coefficients.

Proposition 5. Suppose $Y \stackrel{f}{\rightarrow} S^{n}$ is cohomologically trivial and $H^{n-1} Y=0$. Suppose $n-r<j \leqslant \min (n, 2(n-r)+1)$. Then

$$
\left(\Omega^{r} f\right)^{*}\left(e_{j-1-(n-r)} \cup g_{n-r} \otimes g_{n-r}\right)=\sigma^{r}\left(\mathrm{Sq}_{f}^{j}\left(g_{n}\right)\right)
$$


in $H^{n+j-r-1}\left(\Omega^{r} Y\right)$, where $\mathrm{Sq}_{f}^{j}$ denotes the functional cohomology operation, and $e_{j-1-(n-r)} \cup g_{n-r} \otimes g_{n-r} \in H^{n+j-r-1}\left(\Omega^{r} S^{n}\right)$ is as in [8, 3.1(b)].

Proof. Denote $K\left(\mathrm{Z}_{2}, n\right)$ by $K_{n}$. Let $E$ dienote the fibre of $\mathrm{Sq}^{j}: K_{n} \rightarrow K_{n+j}$. In diagram (6) the liftings $l$ and $l^{\prime}$ are unique and $\left(l^{\prime}\right)^{*}\left(l_{n+j-1}\right)=\operatorname{Sq}_{f}^{j}\left(g_{n}\right)$.

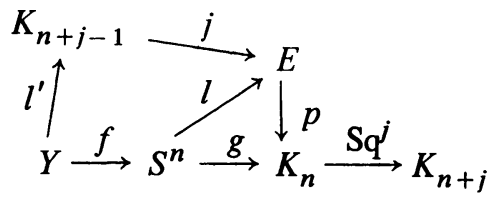

We apply $\Omega^{r}$ to diagram (6) and note that since $\operatorname{Sq}^{j}\left(\iota_{n-r}\right)=0, \Omega^{r} E \simeq K_{n-r} \times$ $K_{n+j-r-1}$.

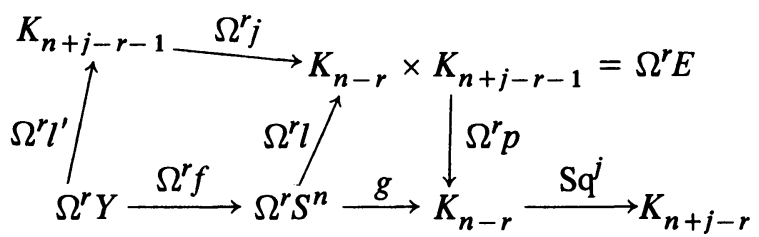

LEMMA 8. In (7), there is $y \in H^{n+j-r-1}\left(\Omega^{r} E\right)$ such that $\left(\Omega^{r} j\right)^{*}(y)=\imath$ and $\left(\Omega^{r} l\right)^{*}(y)=e_{j-1-(n-r)} \cup g_{n-r} \otimes g_{n-r}$.

Thus

$$
\begin{aligned}
\left(\Omega^{r} f\right)^{*}\left(e_{j-1-(n-r)} \cup g_{n-r} \otimes g_{n-r}\right) & =\left(\Omega^{r} f\right)^{*}\left(\Omega^{r} l\right)^{*}(y) \\
& =\left(\Omega^{r} l^{\prime}\right)^{*}(\iota)=\sigma^{r}\left(\operatorname{Sq}_{f}^{j}\left(g_{n}\right)\right) .
\end{aligned}
$$

Proof of Lemma 8. The proof proceeds by induction on $r+j$, beginning with the case $r+j=n+1$. Milgram showed in [7, 3.1.1] that there is $y \in H^{2 n-2 r}\left(\Omega^{r} E\right)$ such that $\Delta(y)=y \otimes 1+1 \otimes y+\left(\Omega^{r} p\right)^{*}(\iota) \otimes\left(\Omega^{r} p\right)^{*}(\iota)$ and $\left(\Omega^{r} j\right)^{*}(y)=\imath$. Then

$$
\begin{aligned}
\left\langle\left(\Omega^{r} l\right)^{*} y, g_{n-r} \otimes g_{n-r}\right\rangle & =\left\langle\Delta y,\left(\Omega^{r} l\right)_{*} g_{n-r} \otimes\left(\Omega^{r} l\right)_{*}\left(g_{n-r}\right)\right\rangle \\
& =\left\langle\Omega^{r} p^{*} l,\left(\Omega^{r} l\right)_{*} g_{n-r}\right\rangle^{2} \neq 0 .
\end{aligned}
$$

Now in the general case, the induction hypothesis provides a class $y_{r-1} \in$ $H^{n+j-r}\left(\Omega^{r-1} E\right)$ such that $\left(\Omega^{r-1} j\right)^{*} y_{r-1}=\imath$ and $\left\langle y_{r-1}\right.$, $\left.\left(\Omega^{r-1} l\right)_{*}\left(Q_{j+r-2-n} g_{n-r-1}\right)\right\rangle \neq 0$, where $Q$ denotes the Dyer-Lashof operation. We let $y_{r}=\sigma y_{r-1}$. Then $\left(\Omega^{r} j\right)^{*} y_{r}=\iota$ and

$$
\begin{aligned}
\left\langle y_{r},\left(\Omega^{r} l\right)_{*}\left(Q_{j+r-1-n} g_{n-r}\right)\right\rangle & =\left\langle\sigma y_{r-1},\left(\Omega^{r} l\right)_{*} \tau\left(Q_{j+r-2-n} g_{n-r+1}\right)\right\rangle \\
& =\left\langle\sigma y_{r-1}, \tau\left(\Omega^{r-1} l\right)_{*} Q_{j+r-2-n} g_{n-r+1}\right\rangle \\
& =\left\langle y_{r-1},\left(\Omega^{r-1} l\right)_{*} Q_{j+r-2-n} g_{n-r+1}\right\rangle \neq 0 .
\end{aligned}
$$

Here $\tau$ denotes the transgression in the appropriate path fibrations. The 
lemma follows because $e_{j-1-(n-r)} \cup g_{n-r} \otimes g_{n-r}$ is the only nonzero class in its dimension.

\section{REFERENCES}

1. J. F. Adams, Vector fields on spheres, Ann. of Math. (2) 75 (1962), 603-632.

2. D. M. Davis, Generalized homology and the generalized vector field problem, Quart. J. Math. 25 (1974), 169-193.

3. Nonexistence of axial maps, Topology and its Applications, Dekker, New York, 1974, pp. 137-141.

4. D. M. Davis and M. Mahowald, Obstruction theory and ko-theory (Proc. 1977 Evanston Conf.), Lecture Notes in Math., Springer-Verlag, Berlin and New York (to appear).

5. S. Feder and W. Iberkleid, Secondary operations in $K$-theory and sections of vector bundles (Geometry and topology, Rio de Janeiro, 1976), Lecture Notes in Math., no. 597, SpringerVerlag, Berlin and New York, 1977, pp. 161-175.

6. M. Mahowald, On the unstable J-homomorphism, Reunion sobre Teoria Homotopia, Notas de Matematicas y Simposia, Soc. Mat. Mexicana, vol. 1, 1975, pp. 99-105.

7. R. J. Milgram, The structure over the Steenrod algebra of some 2-stage Postnikov systems, Quart J. Math. 20 (1969), 161-169.

8. __ Unstable homotopy from the stable point of view, Lecture Notes in Math., no. 368, Springer-Verlag, Berlin and New York, 1974.

9. H. Toda, Composition methods in homotopy groups of spheres, Ann. of Math. Studies, no. 49, Princeton Univ. Press, Princeton, N. J., 1962.

Department of Mathematics, Lehigh University, Bethlehem, Pennsylvania 18015

Department of Mathematics, NorthWestern University, Evanston, Illinois 60201 